\title{
Performance Analysis of Adaptive Neuro Fuzzy Inference System Control for MEMS Navigation System
}

\author{
Ling Zhang, Jianye Liu, Jizhou Lai, and Zhi Xiong \\ Navigation Research Center, Nanjing University of Aeronautics and Astronautics, China \\ Correspondence should be addressed to Ling Zhang; zhanglingsnowman@nuaa.edu.cn
}

Received 21 September 2013; Accepted 31 October 2013; Published 30 January 2014

Academic Editor: Xiaojie Su

Copyright ( 2014 Ling Zhang et al. This is an open access article distributed under the Creative Commons Attribution License, which permits unrestricted use, distribution, and reproduction in any medium, provided the original work is properly cited.

\begin{abstract}
Characterized by small volume, low cost, and low power, MEMS inertial sensors are widely concerned and applied in navigation research, environmental monitoring, military, and so on. Notably in indoor and pedestrian navigation, its easily portable feature seems particularly indispensable and important. However, MEMS inertial sensor has inborn low precision and is impressionable and sometimes goes against accurate navigation or even becomes seriously unstable when working for a period of time and the initial alignment and calibration are invalid. A thought of adaptive neuro fuzzy inference system (ANFIS) is relied on, and an assistive control modulated method is presented in this paper, which is newly designed to improve the inertial sensor performance by black box control and inference. The repeatability and long-time tendency of the MEMS sensors are tested and analyzed by ALLAN method. The parameters of ANFIS models are trained using reasonable fuzzy control strategy, with high-precision navigation system for reference as well as MEMS sensor property. The MEMS error nonlinearity is measured and modulated through the peculiarity of the fuzzy control convergence, to enhance the MEMS function and the whole MEMS system property. Performance of the proposed model has been experimentally verified using low-cost MEMS inertial sensors, and the MEMS output error is well compensated. The test results indicate that ANFIS system trained by high-precision navigation system can efficiently provide corrections to MEMS output and meet the requirement on navigation performance.
\end{abstract}

\section{Introduction}

Characterized by small volume, low cost, and low power, MEMS inertial sensors are widely concerned and applied to navigation research, environmental monitoring, military and so on. Notably in unmanned air systems, its easily portable feature seems particularly indispensable and important.

However, MEMS inertial sensor HAS inborn low precision, and impressionable. It sometimes goes against accurate navigation or even becomes seriously unstable when working for a period of time, and more worse the initial alignment and calibration are invalid. According to this, many scholars use the Kalman filter method to compensate and correct the MEMS error. By this way, the MEMS system performance can be improved, but its effect is not good, as discussed in $[1,2]$.

A thought of adaptive neurofuzzy inference system (ANFIS) is relied on. Compared with fuzzy inference system and artificial neural network, as discussed in [3-6], adaptive neurofuzzy inference system (ANFIS) not only has advantages of the two methods but also makes up for their shortcomings. On one hand, it has effective self-learning mechanism and achieves self-learning function. On the other hand, it has a variety of neural networks, optimizes the control rules, and expresses the reasoning-function like human brain. It makes the system develop towards adaptive, selforganizing and self-learning, as discussed in [7-11].

As Integrated avionics system for vehicle is composed of different kinds of sensors to change the separated state, and achieve complementary, mutual backup and integrated usage information, where the system includes MEMS inertial measurement unit (MEMS-IMU) and high-precision IMU. According to this, ANFIS is newly designed to use reference IMU to improve MEMS-IMU performance by black box control and inference. The MEMS sensor error is measured and modulated through the peculiarity of the fuzzy control convergence, to enhance the MEMS function and the whole 
MEMS system property. Performance of the proposed model is experimentally verified using low-cost MEMS inertial sensors, to meet the requirement on navigation performance.

\section{Analysis of MEMS-IMU Property}

2.1. Error Modelling of MEMS Inertial Sensor. As to error of MEMS-IMU outputs, constant error, scale factor error and installation error, are considered as the main composition of the IMU error. What is more, random error is also inevitable and impacts the output accuracy. According to this, and ignoring more than one-order small amount, the accelerometer model is built as follows, as discussed by the author in [12]:

$$
\begin{aligned}
a_{m} & =\left(I+K_{a}\right)\left(I+\theta_{a}\right) a+\varepsilon_{a}+\nabla a \\
& \approx\left(I+K_{a}+\theta_{a}\right) a+\varepsilon_{a}+\nabla a .
\end{aligned}
$$

Then, the accelerometer error model is

$$
\Delta a=\nabla a+K_{a} a+\theta_{a} a+\varepsilon_{a} .
$$

In calibration, the random error $\varepsilon_{a}$ is mainly affected by temperature. So $\varepsilon_{a}$ may be expressed as

$$
\begin{aligned}
\varepsilon_{a} & =\varepsilon_{a T}+\varepsilon_{a a} \\
& =a_{a T} * \Delta T+b_{a T} *(\Delta T)^{2}+c_{a T} *(\Delta T)^{3}+\varepsilon_{a a} .
\end{aligned}
$$

Similarly, the gyroerror model is

$$
\Delta \omega=\nabla \omega+K_{\omega} \omega+\theta_{\omega} \omega+\varepsilon_{\omega} .
$$

And, the $\varepsilon_{\omega}$ is expressed as

$$
\begin{aligned}
\varepsilon_{\omega} & =\varepsilon_{\omega T}+\varepsilon_{\omega \omega} \\
& =a_{\omega T} * \Delta T+b_{\omega T} *(\Delta T)^{2}+c_{\omega T} *(\Delta T)^{3}+\varepsilon_{\omega \omega},
\end{aligned}
$$

where $\Delta \omega$ and $\Delta a$ are IMU error; $\nabla \omega$ and $\nabla a$ are IMU constant drift; $K_{\omega}$ and $K_{a}$ are the scale factor error matrix; $\theta_{\omega}$ and $\theta_{a}$ are the alignment matrix; $\Delta T$ is temperature variation; $a_{\omega T}, b_{\omega T}$, and $c_{\omega T}$ are the gyroerror coefficients affected by temperature; $a_{a T}, b_{a T}$, and $c_{a T}$ are the accelerometer error coefficients affected by temperature; $\varepsilon_{\omega \omega}$ and $\varepsilon_{a a}$ are the random error.

2.2. Experimental Analysis and Compensation of MEMS Inertial Sensor. The principle of rotating SINS is elaborated as follows. At the beginning, the rotating coordinate $(s)$ is coincident with the body coordinate $(b)$, and $o x_{s}$ represents the real $x$-axis of gyro- and accelerometer; $o y_{s}$ represents the real $y$-axis of gyro-, and accelerometer; $o z_{s}$ is coincident with the $z$-axis of $b$ coordinate and vertical with $o x_{s}, o y_{s}$. The effective IMU outputs are received from the real IMU outputs in the process of coordinate conversion. The rotating angle is

$$
\alpha=\Omega \cdot t
$$

The coordinate transformation matrix from $s$ to $b$ is

$$
\begin{aligned}
C_{s}^{b} & =\left[\begin{array}{ccc}
\cos \theta & -\sin \theta & 0 \\
\sin \theta & \cos \theta & 0 \\
0 & 0 & 1
\end{array}\right] \\
& =\left[\begin{array}{ccc}
\cos (\Omega t) & -\sin (\Omega t) & 0 \\
\sin (\Omega t) & \cos (\Omega t) & 0 \\
0 & 0 & 1
\end{array}\right] .
\end{aligned}
$$

Then, the gyroerror is

$$
\begin{aligned}
& \Delta \omega^{b}=C_{s}^{b} \Delta \omega^{s}=C_{s}^{b}\left(\nabla \omega+K_{\omega} \omega+\theta_{\omega} \omega+\varepsilon_{\omega}\right) \\
& =C_{s}^{b}\left(\left[\begin{array}{l}
\nabla \omega_{x} \\
\nabla \omega_{y} \\
\nabla \omega_{z}
\end{array}\right]+\left[\begin{array}{lll}
K_{\omega x} & & \\
& K_{\omega y} & \\
& & K_{\omega z}
\end{array}\right]\left[\begin{array}{l}
\omega_{x} \\
\omega_{y} \\
\omega_{z}
\end{array}\right]\right. \\
& \left.+\left[\begin{array}{ccc} 
& \theta_{\omega x z} & -\theta_{\omega x y} \\
-\theta_{\omega y z} & & \theta_{\omega y x} \\
\theta_{\omega z y} & -\theta_{\omega z z} &
\end{array}\right]\left[\begin{array}{l}
\omega_{x} \\
\omega_{y} \\
\omega_{z}
\end{array}\right]+\varepsilon_{\omega}\right)
\end{aligned}
$$

Through analysis item by item,

$$
\begin{aligned}
C_{s}^{b} \nabla \omega & =\left[\begin{array}{ccc}
\cos (\Omega t) & -\sin (\Omega t) & 0 \\
\sin (\Omega t) & \cos (\Omega t) & 0 \\
0 & 0 & 1
\end{array}\right]\left[\begin{array}{l}
\nabla \omega_{x} \\
\nabla \omega_{y} \\
\nabla \omega_{z}
\end{array}\right] \\
& =\left[\begin{array}{c}
\nabla \omega_{x} \cos (\Omega t)-\nabla \omega_{y} \sin (\Omega t) \\
\nabla \omega_{x} \sin (\Omega t)+\nabla \omega_{y} \cos (\Omega t) \\
\nabla \omega_{z}
\end{array}\right] .
\end{aligned}
$$

In (9), it is indicated that such constant errors as $\nabla \omega_{x}, \nabla \omega_{y}$, and $\nabla \omega_{z}$ may be modulated by periodic rotation, and the error impact will be smaller.

However, as to error coefficients $K_{\omega}$ and $\theta_{\omega}$, the impact may be partly modulated. The $x$-axis error caused by $K_{\omega}$ and $\theta_{\omega}$ in $b$ coordinate is

$$
\begin{aligned}
C_{s}^{b}\left(K_{\omega} \omega+\theta_{\omega} \omega\right)= & C_{s}^{b}\left(\left[\begin{array}{lll}
K_{\omega x} & & \\
& K_{\omega y} & \\
& & K_{\omega z}
\end{array}\right]\left[\begin{array}{l}
\omega_{x} \\
\omega_{y} \\
\omega_{z}
\end{array}\right]\right. \\
& \left.+\left[\begin{array}{ccc} 
& \theta_{\omega x z} & -\theta_{\omega x y} \\
-\theta_{\omega y z} & & \theta_{\omega y x} \\
\theta_{\omega z y} & -\theta_{\omega z z}
\end{array}\right]\left[\begin{array}{l}
\omega_{x} \\
\omega_{y} \\
\omega_{z}
\end{array}\right]\right) \\
= & {\left[\begin{array}{ccc}
\cos (\Omega t) & -\sin (\Omega t) & 0 \\
\sin (\Omega t) & \cos (\Omega t) & 0 \\
0 & 0 & 1
\end{array}\right] } \\
& \times\left[\begin{array}{c}
K_{\omega x} \omega_{x}+\theta_{\omega x z} \omega_{y}-\theta_{\omega x y} \omega_{z} \\
-\theta_{\omega y z} \omega_{x}+K_{\omega y} \omega_{y}+\theta_{\omega y x} \omega_{z} \\
\theta_{\omega z y} \omega_{x}-\theta_{\omega z z} \omega_{y}+K_{\omega z} \omega_{z}
\end{array}\right] .
\end{aligned}
$$

Set $K_{\omega x} \omega_{x}+\theta_{\omega x z} \omega_{y}-\theta_{\omega x y} \omega_{z}$ as an example; if $K_{\omega x} \omega_{x}+\theta_{\omega x z} \omega_{y}-$ $\theta_{\omega x y} \omega_{z}$ can be expressed as $a+f\left(x_{i}\right), i=1,2,3$, the error impact caused by $a$ may be modulated to zero, where a is the inductive constant and $f\left(x_{i}\right), i=1,2,3$ is the variable part. 
TABLE 1: Means and deviation of MEMS gyro- and accelerometer.

\begin{tabular}{|c|c|c|c|c|c|c|}
\hline & $x$-gyro $(\% / s)$ & $y$-gyro $(\% / s)$ & $z$-gyro $(\% / s)$ & $x$-acce $(\mathrm{m} / \mathrm{s} / \mathrm{s})$ & $y$-acce $(\mathrm{m} / \mathrm{s} / \mathrm{s})$ & $z$-acce $(\mathrm{m} / \mathrm{s} / \mathrm{s})$ \\
\hline \multicolumn{7}{|l|}{ MEMS-IMU raw data } \\
\hline Mean & 4.25 & 9.86 & 9.71 & 0.193 & 0.211 & 0.205 \\
\hline Standard deviation & 0.35 & 0.55 & 0.51 & 0.038 & 0.036 & 0.053 \\
\hline \multicolumn{7}{|c|}{ MEMS-IMU equivalent data } \\
\hline Mean & -0.106 & -0.019 & 10.012 & 0.013 & 0.002 & 0.074 \\
\hline Standard deviation & 7.903 & 7.969 & 4.063 & 0.281 & 0.194 & 0.099 \\
\hline
\end{tabular}

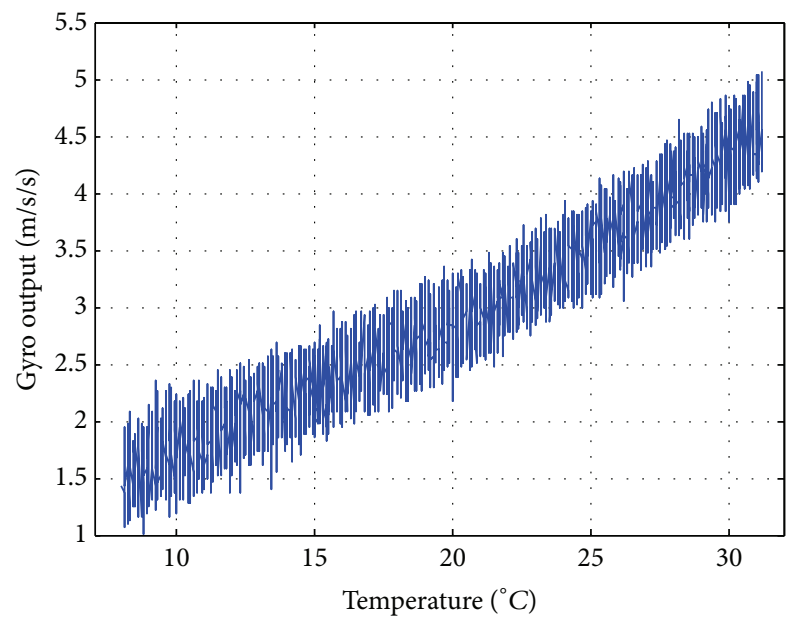

FIGURE 1: Gyro output changed with temperature.

The accelerometer error is

$$
\Delta f^{b}=C_{s}^{b} \Delta f^{s}=C_{s}^{b}\left(\nabla f+K_{f} f+\theta_{f} f+\varepsilon_{f}\right)
$$

The error analysis and modulation are similar to those of gyros.

It is clearly shown from MEMS-IMU raw data in Table 1 that means and deviations of MEMS-IMU are bigger than those of normal situations. Through rotation modulation, mean data indicates that nonstochastic errors of MEMS-IMU are effectively and quickly improved when calculating navigation parameters. However, due to rotation movement by cosine function, the standard deviation is bigger than that of law data.

Moreover, the MEMS outputs are easily affected by environment. Generally, the temperature in use is -40 to $70^{\circ} \mathrm{C}$, and the internal structure of MEMS device may change under different temperature conditions. In such situation, the measured capacitance output of the inertial sensor is deviated from the normal value, and the output of the inertial sensor is inevitably composed of real value and error. Figures 1 and 2 are separately curves of gyro- and accelerometer values changed with temperature.

As the MEMS performance is unstable, the calibration is not always effective due to its temperature impact. When there is $5^{\circ}$ temperature rise, the output will be $10 \%$ changed according to the raw data at room temperature.

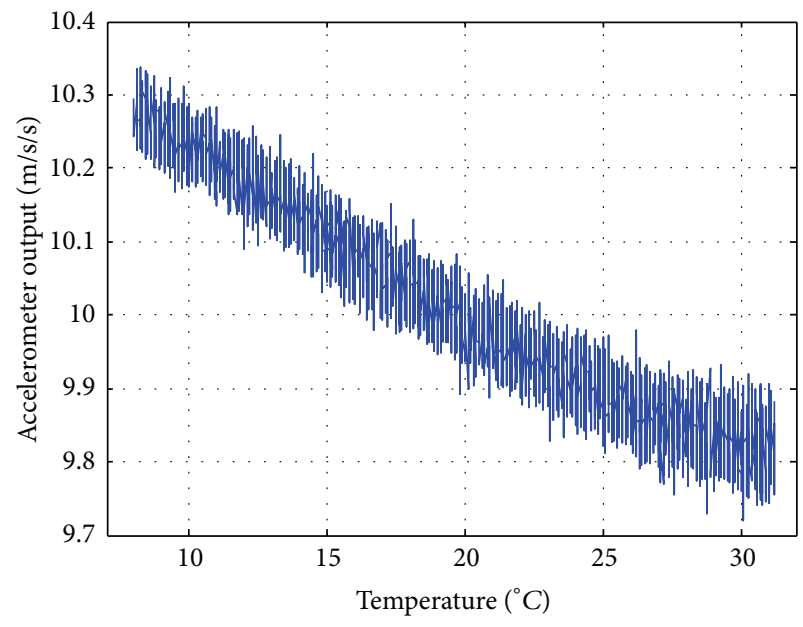

Figure 2: Accelerometer output changed with temperature.

\section{ANFIS Identification and Compensation Program for MEMS-IMU}

3.1. ANFIS Structure. ANFIS is a class of adaptive networks, and it makes the integration of the advantages of neural network and fuzzy inference system. Detailed mathematical progress of ANFIS is as follows. Firstly, it maps the input data by adjusting the shape and parameters of the membership function. Secondly, it remaps the data from the input space to the output space by the membership function of the output variables. During this process, the least squares algorithm is used to adjust ANFIS conclusion parameters, and the gradient descent algorithm is used to adjust ANFIS premise parameters, where the channel for adjusting conclusion parameters is called forward channel and the channel for adjusting the premise parameters is called backward channel, as discussed in [13-16].

In ANFIS structure, as to two input parameters $x, u$ and one output $y$, together with first-order Sugeno fuzzy model, there are two if-then fuzzy rules as follows

The equivalent ANFIS structure is as shown in Figure 3.

In the first layer, each knot is an adaptive knot with function. For example,

$$
O_{1, i}=A_{i}(x), \quad i=1,2,
$$

where $x$ is input of $\operatorname{knot} I$ and $A$ is the relevant fuzzy language identification. 


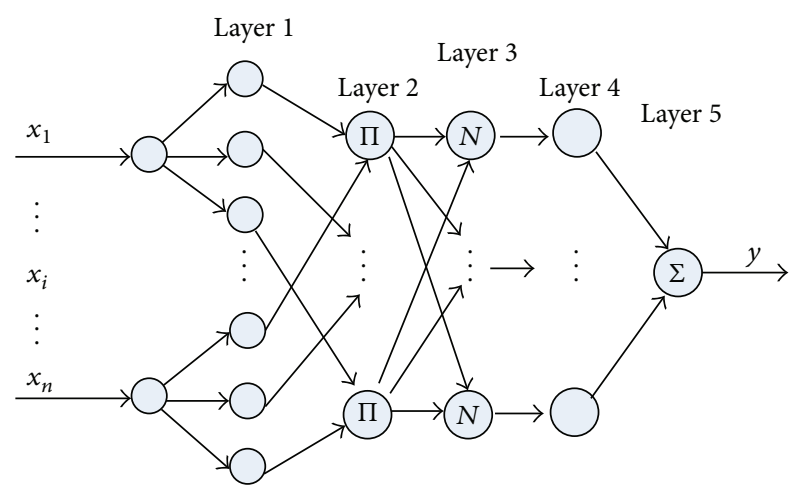

FIGURE 3: Equivalent ANFIS structure.

In the second layer, each knot is a fixed knot identified by $\Pi$. The output is the product of all input signals and represents the firing strength of a rule as

$$
\mathrm{O}_{2, i}=A_{i}(x) \ldots
$$

In the third layer, the ratio of firing strength for rule $i$ and the whole firing strength for all the rules is calculated, which means that the firing strength of each rule is normalized as

$$
O_{3, i}=\bar{\omega}=\frac{\omega_{i}}{\sum \omega}
$$

In the fourth layer, each knot has its own consequent node and output. The $i$ th knot is an adaptive knot with function

$$
O_{4, i}=\overline{\omega_{i}} f_{i}=\overline{\omega_{i}}\left(p_{i} x+q_{i} y+r_{i}\right)
$$

where $\overline{\omega_{i}}$ is normalized motive force transformed from layer 3 and $p 1, q 1, r 1$ are parameters. The parameters in this layer are called conclusion parameters.

In the fifth layer, each knot is a fixed knot identified with $\sum$, and it calculates the output sum by all the signals

$$
O_{5, i}=\sum \overline{\omega_{i}} f_{i}=\frac{\sum \overline{\omega_{i}} f_{i}}{\sum \overline{\omega_{i}}} .
$$

When ANFIS is trained by reduplicative iteration, the pending characteristic parameters are adjusted dynamically to make sure the accuracy identified by ANFIS meets the requirement. The premise parameters and conclusion parameters are received, so that the ANFIS system is determined, as discussed in [17-19].

3.2. ANFIS System Training. As to inputs of ANFIS, the outputs of MEMS-IMU and standard IMU are segmented into different training data spaces. The initial structure of ANFIS is preset with 5 layers, and ANFIS uses the rule firing strength $\omega_{j}(j=1, \ldots, M(K))$ as a criterion for each rule, where $M(k)$ is the number of clusters at $K$. At time $K$, the separate input training data is $x_{i}(i=1, \ldots, n)$, and $n$ is the numbers of inputs.

If $\omega_{j}\left(x_{i}\right) \leq \alpha$ th, the rule is updated into a new one, and $\widetilde{M}=M(k)+1$. Random $\alpha$ th $\in(0.1,0.5)$ is a preset threshold, it values $\omega_{j}$ and provides reference for new cluster generated in ANFIS. If $\alpha$ th is smaller, the number of clusters is larger, and the fuzzy system is more complicated.

When $\widetilde{M}$ is updated, it means that new cluster is generated, and needs to rebuild fuzzy system. The selected cluster centre may be set a bigger weight coefficient. This progress is repeatedly taken with inputs data, until the ANFIS meets the accuracy requirements.

ANFIS's parameters include premise parameters and consequent parameters. They are updated based on gradient descent learning algorithm. When the premise parameters are fixed, the least squares algorithm is applied to calculate the consequent data.

This is the whole progress of ANFIS(i) generation. In order to speed up the ANFIS progress and enhance the precision, all the data are separated into different parts for training and checking. The prior ANFIS structure is valued by the checking data and set as premise structure for next system generation, up to the expected modulated effect. The proposed ANFIS training process is shown as in Figure 4.

3.3. MEMS-IMU Error Compensation Based ANFIS. In integrated avionics system for vehicle, synthesis of multiple sensors is a trend; different kinds of sensors may change the separated state and achieve complementary, mutual-backup and integrated usage information provided by each sensor. Through integrated control and management of multisensors, the sensor system may have higher performance level than that of any single sensor.

Generally, as a backup system, MEMS inertial navigation system has low performance by comparison. In order to improve it, high-precision INS output is applied to help correcting MEMS-IMU outputs by ANFIS method. Highprecision INS outputs are set as standard information and separated into different spaces together with MEMS-IMU outputs. The different data spaces are divided into training parts and checking parts. The 3D outputs of MEMS gyros and accelerometers are trained objects and inputs of ANFIS. ANFIS works in update mode; the structure is built, valued, and screened continuously, until the optimal structure meets accuracy demands. The ANFIS building program for MEMSIMU is shown as in Figure 5.

When standard IMU outputs are lacked, ANFIS structure switches to the correction mode. The identified and trained error corrector by ANFIS is applied to modify the law MEMSIMU output and help provide higher-precision MEMS-IMU outputs.

\section{Experiment and Analysis}

The MEMS-IMU used in this paper is MPU6050. The MEMS gyro drift is $4.25(\% / \mathrm{s})$, and random is $0.35\left(^{\circ} / \mathrm{s}\right)$; MEMS accelerometer bias is $0.193 \mathrm{~m} / \mathrm{s} / \mathrm{s}$, and random is $0.038 \mathrm{~m} / \mathrm{s} / \mathrm{s}$. The output frequency of MEMS-IMU is $50 \mathrm{~Hz}$. As to standard IMU, the gyro drift is $0.003(\%)$, and random is $0.003(\%)$; the accelerometer bias is $0.043 \mathrm{~m} / \mathrm{s} / \mathrm{s}$, random is $0.017 \mathrm{~m} / \mathrm{s} / \mathrm{s}$, and the output frequency is $50 \mathrm{~Hz}$ too. 


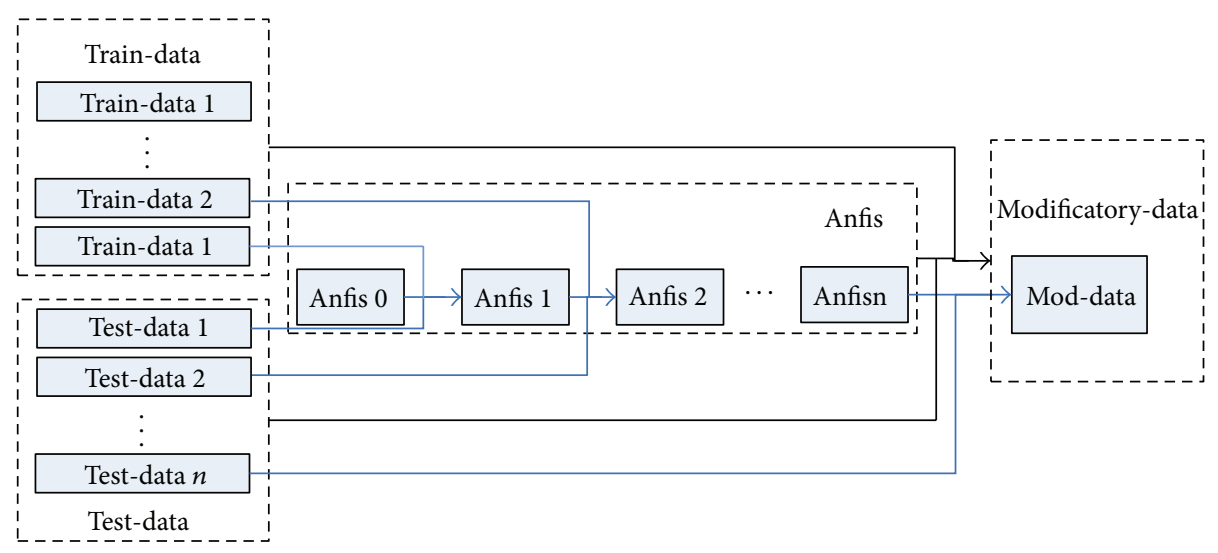

FIGURE 4: ANFIS training-process program.

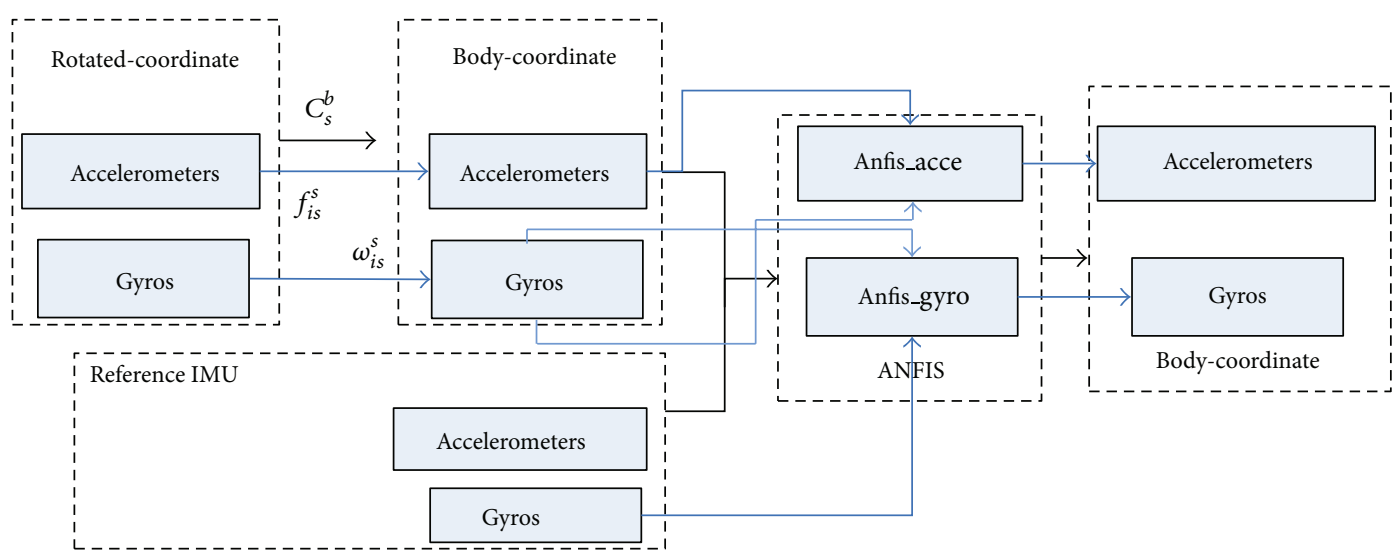

Figure 5: ANFIS building program for MEMS-IMU.

TABLE 2: Bias of IMU data.

\begin{tabular}{lcccccc}
\hline & $x$-gyro & $y$-gyro & $z$-gyro & $x$-acce & $y$-acce & $z$-acce \\
\hline MEMS-IMU data & 4.251 & -9.86 & -9.71 & 0.193 & 0.211 & 0.187 \\
Standard IMU data & 0.001 & -0.003 & 0.002 & -0.043 & 0.014 & 0.026 \\
Modified IIMU data & 0.001 & -0.003 & 0.002 & -0.043 & 0.015 & 0.027
\end{tabular}

TABle 3: Random of IMU data.

\begin{tabular}{lcccccc}
\hline & $x$-gyro & $y$-gyro & $z$-gyro & $x$-acce & $y$-acce & $z$-acce \\
\hline MEMS-IMU data & 0.352 & 0.553 & 0.516 & 0.038 & 0.036 & 0.053 \\
Standard IMU data & 0.003 & 0.003 & 0.004 & 0.065 & 0.046 & 0.017 \\
Modified IIMU data & 0.002 & 0.041 & 0.003 & 0.121 & 0.027 & 0.012 \\
\hline
\end{tabular}

In order to testify the effectiveness of ANFIS modulation for MEMS-IMU performance under the auxiliary of highprecision standard IMU, both MEMS-IMU and standard IMU start simultaneously; then corresponding data is sent for training and testing. From Figures 6, 7, and 8, it is clear that MEMS-IMU data is modified, and the precision is highly improved. Tables 2 and 3 show the specific improved level of IMU data by drift and random quantity. Simultaneously, compared with random of each standard gyro, that of tested gyro in Table 2 also indicates that the tested data will be

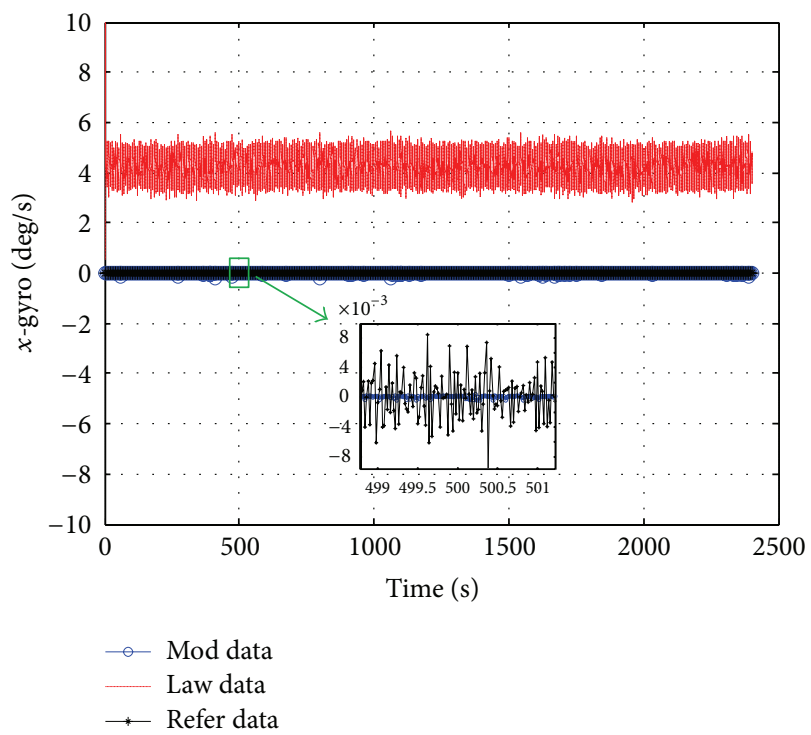

FIgURE 6: Comparison of each $x$-gyro data.

modified towards the standard data, and not only that, but also stability and smoothness of the modified data will be better than those of standard data. Where, blue line with ring 


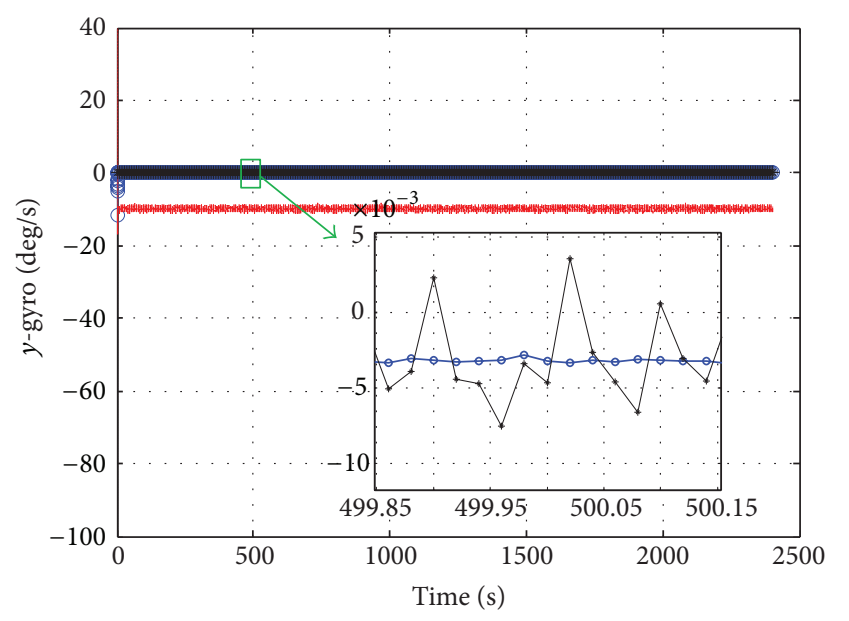

$\rightarrow \quad$ Mod data
$\rightarrow-\ldots$ Law data
$\rightarrow \quad$ Refer data

Figure 7: Comparison of each $y$-gyro data.

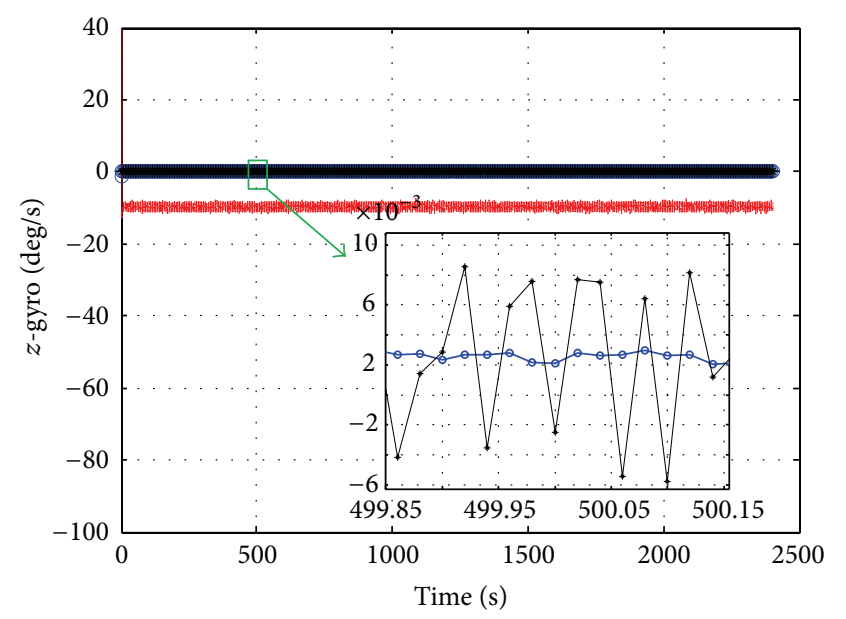

Mod data
- Law data
* Refer data

Figure 8: Comparison of each $z$-gyro data.

represents modified MEMS-IMU data by ANFIS, red line represents MEMS-IMU data to be tested, and black line with star represents standard IMU data with high precision.

To be sure, the effect of modified accelerometer data is not as good as that of gyro, just because the standard accelerometer precision is not so high, and the modified effect has nothing to do with ANFIS structure. In other words, the performance level of standard data may be one of the main influence factors to decide the performance level of the modified data.

\section{Conclusions}

MEMS-IMU is characterized by its small size, low cost, and easy integration, so it has a wild range of applications.
However, low precision is a stumbling block to MEMS sensor, and sometimes it cannot meet the performance requirements. Nowadays, integrated avionics system for vehicle composes different kinds of sensors to change the separated state and achieve complementary, mutual backup and integrated usage information provided by each sensor. So, high-accuracy IMU may be selected to modify MEMS-IMU performance by high-level outputs.

ANFIS system combines the advantages of neural network and fuzzy control methods, and it is suitable for controlling objects with characters of fuzziness, uncertainty, nonlinearity, and time varying. As to MEMS-IMU property, ANFIS reference program is improved, the reference structure is continuously built for good modification performance, and MEMS-IMU performance is corrected. The experimental results show that ANFIS structure is much closer to the accurate model by multiple establishments, and MEMS-IMU outputs are high-level corrected by rules. Simultaneously, the MEMS-IMU performance is of smoothness by ANFIS modulation. In a word, the updated ANFIS program proposed in this paper is helpful to modify and improve the MEMS-IMU property and is a great reference to enhance MEMS sensor performance.

\section{Conflict of Interests}

The authors declare that there is no conflict of interests regarding the publication of this paper.

\section{Acknowledgments}

This research was funded by the National Natural Science Foundation of China (61174197, 91016019, and 61374115) and the Central University Research Funding (NN2012097, NZ2012003).

\section{References}

[1] W. N. Chen, Q. H. Zeng, R. B. Li, and J. Y. Liu, "Mixed linear regression temperature compensation method for annularvibrating MEMS gyroscope," Journal of Chinese Inertial Technology, vol. 20, no. 1, pp. 99-103, 2012.

[2] H. Qin, L. Cong, and X. Sun, "Accuracy improvement of GPS/ MEMS-INS integrated navigation system during GPS signal outage for land vehicle navigation," Journal of Systems Engineering and Electronics, vol. 23, no. 2, pp. 256-264, 2012.

[3] S. Bououden, M. Chadli, F. Allouani, and S. Filali, "A new approach for fuzzy predictive adaptive controller design using particle swarm optimization algorithm," International Journal of Innovative Computing, Information and Control, vol. 9, no. 9, pp. 3741-3758, 2013.

[4] H. Wang, B. Chen, and C. Lin, "Adaptive neural tracking control for a class of stochastic nonlinear systems with unknown deadzone," International Journal of Innovative Computing, Information and Control, vol. 9, no. 8, pp. 3257-3269, 2013.

[5] Z. Wu, P. Shi, H. Su, and J. Chu, "Exponential synchronization of neural networks with discrete and distributed delays under time-varying sampling," IEEE Transactions on Neural Networks and Learning Systems, vol. 23, no. 9, pp. 1368-1376, 2012. 
[6] H. H. Chiang, Y. L. Chen, and T. T. Lee, "Multi-Stage with neuro-fuzzy approach for efficient on-road speed sign detection and recognition," International Journal of Innovative Computing, Information and Control, vol. 9, no. 7, pp. 2919-2939, 2013.

[7] L. Wu, W. X. Zheng, and H. Gao, "Dissipativity-based sliding mode control of switched stochastic systems," IEEE Transactions on Automatic Control, vol. 58, no. 3, pp. 785-793, 2013.

[8] L. Wu, X. Su, and P. Shi, "Output feedback control of Markovian jump repeated scalar nonlinear systems," IEEE Transactions on Automatic Control, 2013.

[9] X. Su, P. Shi, L. Wu, and Y. D. Song, "A novel control design on discrete-time Takagi-Sugeno fuzzy systems with time-varying delays," IEEE Transactions on Fuzzy Systems, vol. 20, no. 4, pp. 655-671, 2013.

[10] X. Su, P. Shi, L. Wu, and S. K. Nguang, "Induced 2 filtering of fuzzy stochastic systems with time-varying delays," IEEE Transactions on Cybernetics, vol. 43, no. 4, pp. 1251-1264, 2013.

[11] X. Su, L. Wu, and P. Shi, "Senor networks with random link failures: distributed filtering for T-S fuzzy systems," IEEE Transactions on Industrial Informatics, vol. 9, no. 3, pp. 1739-1750, 2013.

[12] L. Zhang, J. Z. Lai, J. Y. Liu, and P. Lü, "Analysis of un-coincide coordinate error in single-axis rotating fiber optic strap-down inertial navigation system," Transactions of Nanjing University of Aeronautics and Astronautics, vol. 28, no. 2, pp. 199-205, 2011.

[13] R. Yang, Z. Zhang, and P. Shi, "Exponential stability on stochastic neural networks with discrete interval and distributed delays," IEEE Transactions on Neural Networks, vol. 21, no. 1, pp. 169-175, 2010.

[14] W. Abdel-Hamid, A. Noureldin, and N. El-Sheimy, "Adaptive fuzzy prediction of low-cost inertial-based positioning errors," IEEE Transactions on Fuzzy Systems, vol. 15, no. 3, pp. 519-529, 2007.

[15] A. Karami, S. Keiter, and H. Hollert, "Fuzzy logic and adaptive neuro-fuzzy inference system for characterization of contaminant exposure through selected biomarkers in African catfish," Environmental Science and Pollution Research, vol. 20, no. 3, pp. 1586-1595, 2012.

[16] Y. P. Liao and S. S. Lin, "A meshless method using the TakagiSugeno fuzzy model," Journal of Engineering Mathematics, vol. 72, pp. 159-175, 2012.

[17] A. Salunkhe and M. Chavan, "Analysis of Adaptive neurofuzzy inference system in different environmental conditions," in Proceedings of the AASRI Conference on Power and Energy Systems (PES '12), pp. 87-92, Hong Kong, China, 2012.

[18] R. Singh, A. Kainthola, and T. N. Singh, "Estimation of elastic constant of rocks using an ANFIS approach," Applied Soft Computing Journal, vol. 12, no. 1, pp. 40-45, 2012.

[19] K. Yetilmezsoy, M. Fingas, and B. Fieldhouse, "An adaptive neuro-fuzzy approach for modeling of water-in-oil emulsion formation," Colloids and Surfaces A, vol. 389, no. 1-3, pp. 50-62, 2011. 


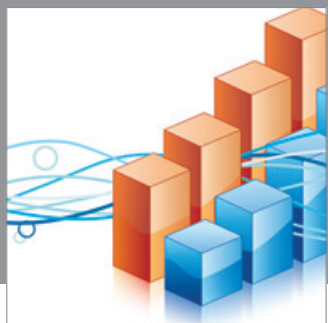

Advances in

Operations Research

mansans

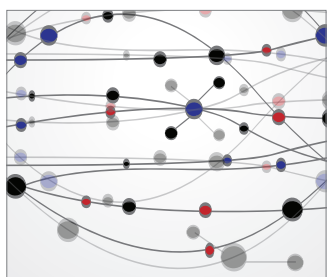

The Scientific World Journal
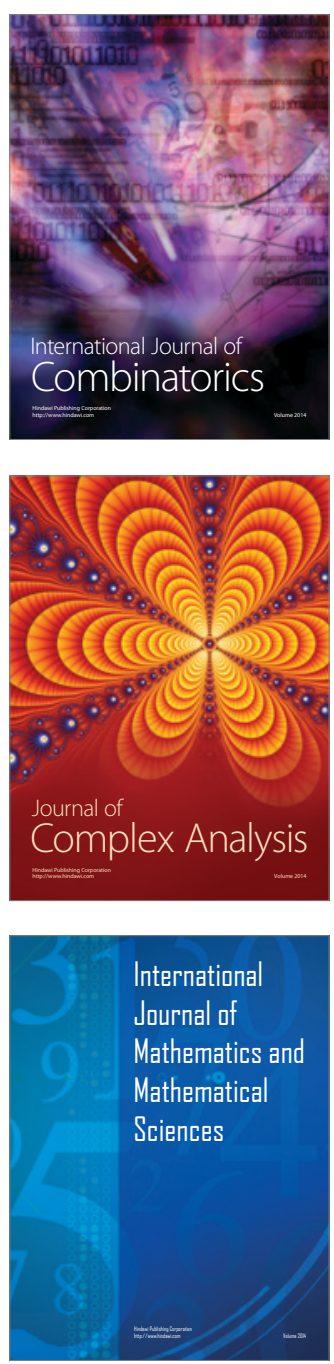
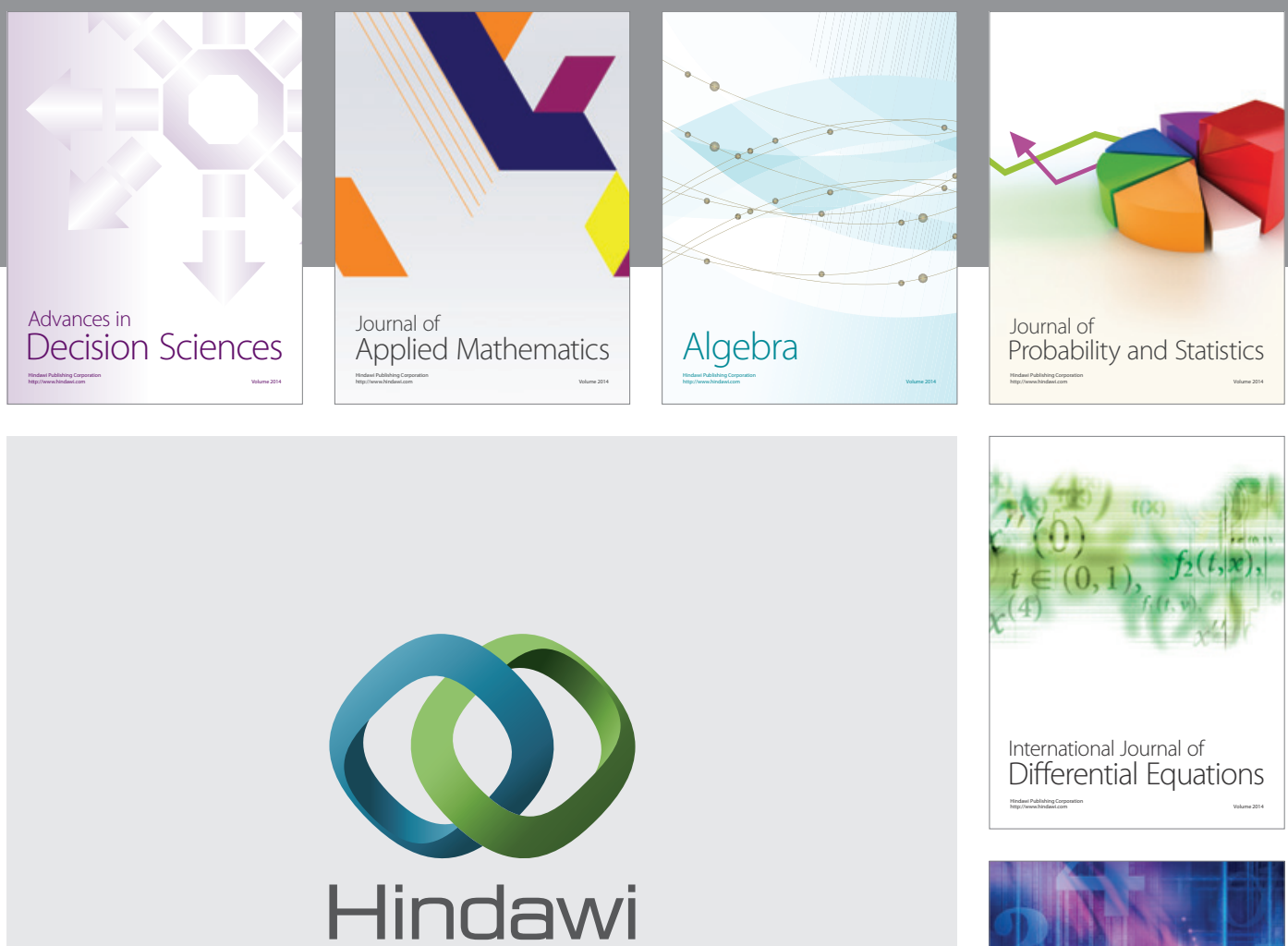

Submit your manuscripts at http://www.hindawi.com
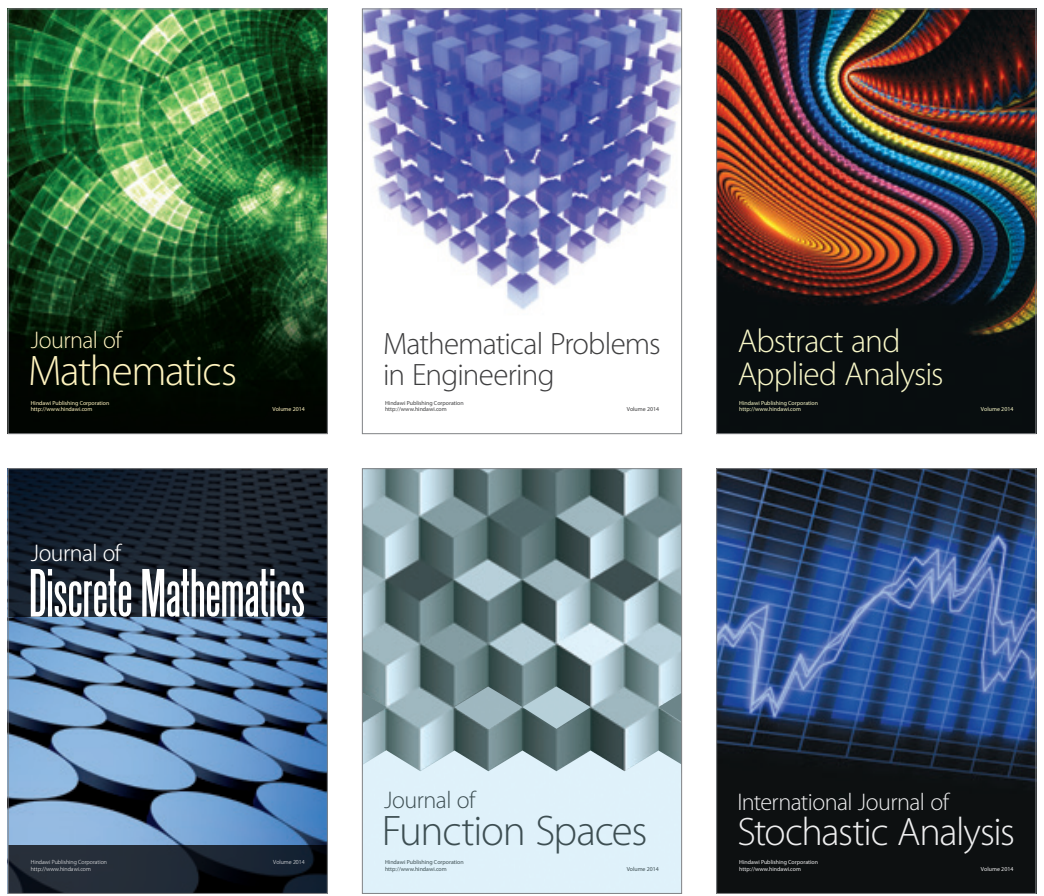

Journal of

Function Spaces

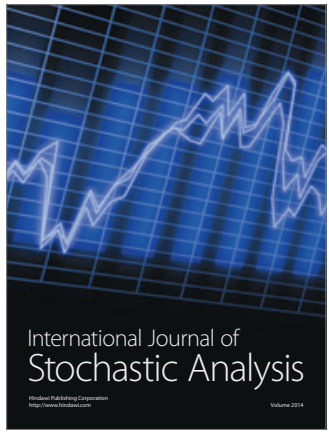

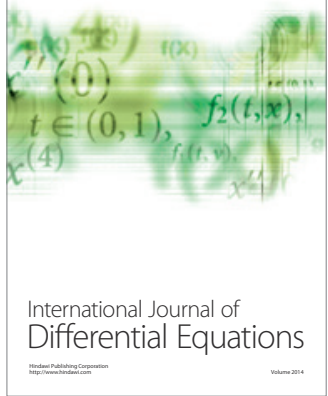
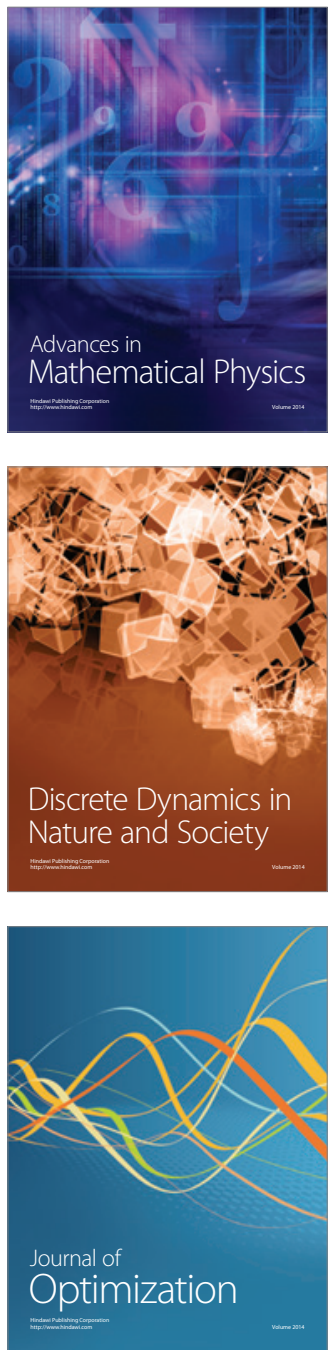